International Journal of Biomedicine I June 2019 - Volume 9, Issue Suppl_1: Abstracts From the Second Russian International Conference "Cryo-electron microscopy 2019: achievements and prospects"

POSTER ABSTRACT PRESENTATIONS

SESSION TITLE: STRUCTURE OF MEMBRANE PROTEINS

DOI: 10.21103/IJBM.9.Suppl_1.P7

\title{
Abstract P-7: Cryoelectron Microscopy Study of Water-Soluble Extracellular Domain of $\alpha 7$ Nicotinic Acetylcholine Receptor
}

Andrey V. Tsarev ${ }^{1,2}$, Roman A. Kamyshinsky ${ }^{3}$, Vasiliy I. Mikirtumov ${ }^{4}$, Dmitriy S. Kulbatskii ${ }^{1}$, Eugene O. Yablokov $^{5}$, Zakhar O. Shenkarev ${ }^{1,2}$, Olga S. Sokolova ${ }^{4}$, Ekaterina N. Lyukmanova ${ }^{1,2}$

${ }^{1}$ Shemyakin-Ovchinnikov Institute of Bioorganic Chemistry, Moscow, Russia; ${ }^{2}$ Moscow Institute of Physics and Technology, Dolgoprudny, Moscow Region, Russian Federation; ${ }^{3}$ National Research Center, Kurchatov Institute, Moscow, Russia; ${ }^{4}$ Lomonosov Moscow State University, Moscow, Russia; ${ }^{5}$ Institute of Biomedical Chemistry, Moscow, Russia

Background: Nicotinic acetylcholine receptor (nAChR) is a ligand-gated ion channel, which is widely distributed both in the central and peripheral nervous system, and in some of the non-neuronal tissues, including epithelium and immune cells. The pathophysiology of a number of diseases is associated with dysfunctions of this receptor, including neurodegenerative and mental disorders like Alzheimer disease (AD) and schizophrenia. The nicotinic receptor of $\alpha 7$ type $(\alpha 7-n A C h R)$ plays important role in the memory and learning processes and is inhibited by soluble aggregates of $\beta$-amyloid peptide $(A \beta) . A \beta_{1-42}$ is the most toxic form of the amyloid peptide.

Methods: The water-soluble analogue of the ligand-binding extracellular domain of $\alpha 7-n A C h R$ was produced in Pichia pastoris and purified from a culture medium by Ni-NTA and size-exclusion chromatography. The equilibrium dissociation constant $(\mathrm{Kd})$ of the complex of the recombinant domain with $\alpha$-bungarotoxin was measured on the optical SPR biosensor Biacore 3000. Structures of the $\alpha 7$ domain alone and in the complex with oligomeric $A \beta_{1-42}$ peptide were studied by cryoelectron microscopy (cryo-EM).

Results: The $\alpha 7$ ligand-binding domain has an increased stability in solution, and demonstrates ligand-binding characteristics similar to those of the native receptor ( $\mathrm{Kd}$ of the domain/ $\alpha$-bungarotoxin complex $28 \pm 2 \mathrm{nM}$ ). Statistical analysis of the cryo-EM images of the individual domain particles revealed the presence of a pentameric structure, confirming intact subunit assembly. Unfortunately, the domain demonstrated the preferable orientation on grids with the top view. Nevertheless, the 3D structure of the domain with a height $\sim 7 \mathrm{~nm}$, external diameter of $\sim 9 \mathrm{~nm}$, and the pore diameter of $\sim 2 \mathrm{~nm}$ was reconstructed at $8.5 \AA$ resolution. 2D classification of the cryo-EM images of the domain particles in the 
complex with $A \beta_{1-42}$ revealed the conformational changes appeared due to interaction with the amyloid peptide.

Conclusion: Obtained results open new perspectives for structural studies of the nAChR ligandbinding domains in complex with the ligands which escape crystallization.

Key Words: cryo-electron microscopy $\bullet \alpha 7-n A C h R \bullet$ beta-amyloid peptide

Sources of Funding: This work was supported by the Russian Science Foundation (project \# 19-7420163).

International Journal of Biomedicine. 2019;9 Suppl 1: S19. doi: 10.21103/IJBM.9.Suppl_1.P7

(C)2019 International Medical Research and Development Corporation 